PROCEEDINGS OF THE

AMERICAN MATHEMATICAL SOCIETY

Volume 66, Number 2, October 1977

\title{
NONOSCILLATION AND EVENTUAL DISCONJUGACY
}

\author{
URI ELIAS
}

\begin{abstract}
If every solution of an $n$th order linear differential equation has only a finite number of zeros in $[0, \infty)$, it is not generally true that for sufficiently large $c, c>0$, every solution has at most $n-1$ zeros in $[c, \infty)$. Settling a known conjecture, we show that for any $n$, the above implication does hold for a special type of equation, $L_{n} y+p(x) y=0$, where $L_{n}$ is an $n$th order disconjugate differential operator and $p(x)$ is a continuous function of a fixed sign.
\end{abstract}

Introduction. An $n$th order linear differential equation

$$
y^{(n)}+p_{1}(x) y^{(n-1)}+\cdots+p_{n}(x) y=0
$$

is said to be disconjugate on an interval $E$ if every one of its nontrivial solutions has at most $n-1$ zeros in $E$ (counting multiplicities). The equation is called nonoscillatory on $E$ if every solution has only a finite number of zeros in $E$.

Assume that every solution of (1) has only a finite number of zeros in $E=[0, \infty)$. Does there exist a half line $[a, \infty), a>0$, in which every solution of (1) has at most $n-1$ zeros? That is, does nonoscillation imply eventual disconjugacy. Generally, the answer is negative [4]. However, this statement holds for special types of equations, e.g.,

$$
\begin{aligned}
\left.\left(r y^{\prime \prime}\right)\right)^{\prime \prime}+p y & =0, \quad[5] \\
y^{(n)}+p y & =0 \quad \text { for even } n,[9] .
\end{aligned}
$$

We prove that nonoscillation implies eventual disconjugacy for a larger family of equations. Consider

$$
L_{n} y+p(x) y=0,
$$

where $L_{n}$ is an $n$th order disconjugate differential operator and $p(x)$ is a continuous function of a fixed sign on $[0, \infty)$ for an arbitrary $n$.

THEOREM 1. If equation (2) is nonoscillatory on $[0, \infty)$, it is eventually disconjugate.

Theorem 1 settles a well-known conjęcture [7].

The $i$ th conjugate point, $\eta_{i}(a)$, is defined as the infimum of the values $b$, such that there exists a solution of (1) which vanishes at $a$ and has at least $n-1+i$ zeros in $[a, b]$. In particular, $\eta_{1}(a) \equiv \eta(a)$ is the infimum of values

Received by the editors January 13, 1977 and, in revised form, April 18, 1977.

AMS (MOS) subject classifications (1970). Primary 34C10.

Key words and phrases. Nonoscillation, eventual disconjugacy, conjugate points. 
$b$, such that (1) is not disconjugate on $[a, b]$.

THEOREM 2. For equation (2), the following properties are equivalent:

(1) The equation has a solution with infinitely many zeros in $[0, \infty)$.

(2) $\eta_{1}(a)$ exists for every $a$.

(3) For a fixed $c, \eta_{i}(c)$ exists for every $i$.

Similar results have been proved in [3] and [10] for (1) under the assumption that no solution has on $[0, \infty)$ a set of zeros of multiplicities 1 , $1, \ldots, 1$ ( $q-1$ times), $2,1,1, \ldots, 1(n-q-1$ times) respectively, and some more assumptions. However, equations of type (2) do not necessarily satisfy this assumption for $n \geqslant 5$. For, choose $k, 1 \leqslant k \leqslant n-3$, such that $(-1)^{n-k} p(x)<0$ and denote by $y_{\lambda}$ the eigenfunction of $L_{n} y+\lambda p(x) y=0$ which corresponds to the $n$ boundary conditions $L_{t} y(a)=0, t=0, \ldots, k-$ $1, L_{0} y(b)=L_{1} y(b)=0, L_{t} y(c)=0, t=0, \ldots, n-k-3, a<b<c$. Given two arbitrary numbers $l, m$, then for a sufficiently large positive eigenvalue $\lambda$, the eigenfunction $y_{\lambda}$ has at least $l$ and $m$ simple zeros in $(a, b)$ and $(b, c)$ respectively [2, Theorem 2$]$.

Proofs. It is well known that a disconjugate operator $L_{n}$ can be written as

$$
L_{n} y=\rho_{n}\left(\rho_{n-1} \cdots\left(\rho_{1}\left(\rho_{0} y\right)^{\prime}\right)^{\prime} \ldots\right)^{\prime},
$$

where $\rho_{i}>0, \rho_{i} \in C^{n-i}, i=0, \ldots, n$. The factorization (3) is not unique. It is known [11], that $\rho_{1}, \ldots, \rho_{n-1}$ can be chosen so that they satisfy

$$
\int^{\infty} \frac{d x}{\rho_{i}(x)}=\infty, \quad i=1, \ldots, n-1 .
$$

In the following, we assume that $L_{n}$ has the representation (3) which satisfies (4). We denote

$$
L_{0} y=\rho_{0} y, \quad L_{i} y=\rho_{i}\left(L_{i-1} y\right)^{\prime}, \quad i=1, \ldots, n .
$$

$L_{0} y, \ldots, L_{n} y$ are called the quasi-derivatives of $y$.

Let $y$ be a solution of (2). We arrange the $n$ quasi-derivatives $L_{0} y, \ldots, L_{n-1} y$ in a cyclic order, so that $L_{n-1} y$ is followed by $L_{0} y$ (cf. [1]). Let $a \leqslant x_{1} \leqslant \cdots \leqslant x_{r}=b$ be the zeros of $L_{0} y, \ldots, L_{n-1} y$ in a certain interval, so that common zeros of consecutive quasi-derivatives will be considered as multiple zeros, but distinct subscripts will be used for zeros of nonconsecutive quasi-derivatives at the same point. Let the number of consecutive quasi-derivatives which vanish at $x_{i}$ be denoted by $n\left(x_{i}\right)$. The total number of (not necessarily consecutive) quasi-derivatives of $y$ which vanish at a point $c$ will be denoted by $\nu(c)$. Obviously, $\nu(c)=\sum_{x_{i}=c} n\left(x_{i}\right)$. In Lemma 1 of [1], the following restriction about the distribution of the zeros of $L_{0} y, \ldots, L_{n-1} y$ is proved:

LEMMA 1. Every solution of (2) satisfies

$$
N(y) \stackrel{\text { def }}{\equiv} \sum_{I} n\left(x_{i}\right)+\sum_{J}\left[n\left(x_{j}\right)-1\right] \leqslant n,
$$


where

$$
\begin{aligned}
& I=\left\{i \mid x_{i}=a \text { or } x_{i}=b \text { or } n\left(x_{i}\right) \text { is even }\right\}, \\
& J=\left\{j \mid a<x_{j}<b \text { and } n\left(x_{j}\right) \text { is odd }\right\} .
\end{aligned}
$$

If. $N(y)=n$, then $\nu(b)$ and $n-\nu(a)$ are both even if $p(x)<0$ and both odd if $p(x)>0$.

Proof of Theorem 1. Assume that (2) is not eventually disconjugate, i.e., $\eta(a) \equiv \eta_{1}(a)$ exists for every $a$. It is well known [6], [8] that there exists an appropriate solution of (2) which has zeros of multiplicities $k$ and $n-k$, $1 \leqslant k \leqslant n-1$, at $a$ and $\eta(a)$ respectively and does not vanish on $(a, \eta(a)) . k$ depends on $a$ and it is not necessarily unique. However, $n-k$ is even if $p(x)<0$ and odd if $p(x)>0$, i.e.,

$$
(-1)^{n-k} p(x)<0 \text {. }
$$

Since only a finite number of values of $k$ is possible, we choose a sequence $\left\{a_{i}\right\}, a_{i} \rightarrow \infty$, so that for each $a_{i}$ corresponds the same index $k=k\left(a_{i}\right)$. For this $k$ and an arbitrary $c$, let $y(x, s)$ be the solution of (2) which satisfies

$$
\begin{aligned}
L_{t} y(c) & =0, & t & =0, \ldots, k-1, \\
L_{t} y(s) & =0, & t & =0, \ldots, n-k-2, \quad s>c .
\end{aligned}
$$

$y(x, s)$ is essentially unique. If there are two independent solutions which satisfy (7)-(8), then there is a linear combination $y$, which has an additional quasi-derivative that vanishes at $c$. This $y$ satisfies $\nu(c) \geqslant k+1, \nu(s) \geqslant n-$ $k-1$ and $N(y) \leqslant n$, hence $\nu(c)=k+1, \nu(s)=n-k-1$. So, by (6), $\nu(s)$ is odd if $p(x)<0$ and even if $p(x)>0$, in contradiction of Lemma 1 .

We prove certain properties of $y(x, s)$. First, no quasi-derivative of $y(x, s)$, except those which are given in (7), can vanish at $x=c$. For $\nu(c) \geqslant k+1$, $\nu(s) \geqslant n-k-1$ is impossible according to the above argument.

$L_{t} y(x, s)$ may have only simple zeros in $(c, s)$. Otherwise, we would have $N(y(x, s)) \geqslant k+2+(n-k-1)>n$, that contradicts (5).

Let $\left\{u_{1}, \ldots, u_{n}\right\}$ be an independent set of solutions of (2). Then $y(x, s)$ is essentially given by the determinant

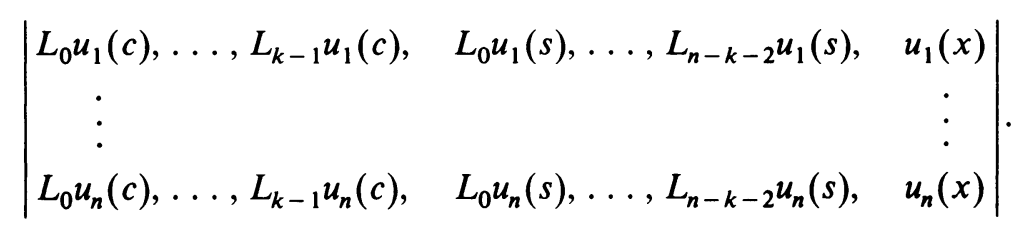

Of course, the determinant is a solution of (2) which satisfies (7) and (8). We have only to show that it is not the trivial solution. If it were identically zero, the submatrix consisting of the first $n-1$ columns would be of rank less than $n-1$. This would contradict the uniqueness proved before of the solution which satisfies (7) and (8). By this representation and the implicit functions theorem it follows that the simple zeros of $L_{t} y(x, s), t=0, \ldots, n-1$, are differentiable functions of $s$. 
By the former stated properties of $y(x, s)$ we conclude, that as $s$ varies, two simple zeros of $L_{t} y(x, s)$ cannot meet in $(c, s)$ and no zero can meet the endpoint $c$. So, since the zeros of $L_{t} y(x, s)$ are differentiable functions of $s$, their number can vary, as $s$ varies, only when a simple zero enters or leaves $(c, s)$ through the endpoint $s$.

We shall prove that as $s \rightarrow \infty$, the number of zeros of $y(x, s)$ in $(c, s)$ tends to infinity and each of these zeros is a bounded function of $s$. This will imply that the limit function $\lim _{s \rightarrow \infty} y(x, s)$ has infinitely many zeros in $[c, \infty)$.

Assume that as $s \rightarrow \infty, L_{0} y(x, s), \ldots, L_{n-1} y(x, s)$ have only a finite number of zeros, if any, in $(c, s)$, which are bounded from above as functions of $s$. Let $M-1$ be their common upper bound. The other zeros (if any) are unbounded. Let $\left\{s_{i}\right\}$ be such a sequence that the unbounded zeros of $L_{0} y(x$, $s_{i}$ ) tend to infinity as $s_{i} \rightarrow \infty$. By Rolle's theorem, the unbounded zeros of $L_{1} y\left(x, s_{i}\right), \ldots, L_{n-1} y\left(x, s_{i}\right)$ also tend to infinity. We normalize the solutions $y\left(x, s_{i}\right)$ by

$$
\sum_{t=0}^{n-1}\left[L_{t} y\left(M, s_{i}\right)\right]^{2}=1 .
$$

The so-normalized family $\left\{y\left(x, s_{i}\right)\right\}$ is a compact set of solutions and it is possible to choose a subsequence $\left\{s_{i_{j}}\right\}, s_{i_{j}} \rightarrow \infty$, and a nontrivial solution $v$ so that $L_{t} y\left(x, s_{i j}\right) \rightarrow L_{t} v, t=0, \ldots, n$, uniformly on compact intervals.

The zeros of $L_{0} y\left(x, s_{i_{j}}\right), \ldots, L_{n-1} y\left(x, s_{i_{j}}\right)$ in $\left[M, s_{i_{j}}\right)$ (if any) tend to infinity as $s_{i j} \rightarrow \infty$ and their other zeros are in $(c, M-1]$. Hence $L_{0} y(x$, $\left.s_{i}\right), \ldots, L_{n-1} y\left(x, s_{i j}\right)$ do not vanish on an arbitrary large right neighborhood of $M-1$, provided $s_{i, j}$ is sufficiently large. Therefore, the quasi-derivatives of $v=\lim _{s_{i j} \rightarrow \infty} y\left(\dot{x}, s_{i j}\right)$ do not change their signs on $(M-1, \infty)$. So they are monotone on $(M-1, \infty)$ and in fact they do not vanish on $[M, \infty)$.

Let $q$ be the greatest index, $1 \leqslant q \leqslant n$, so that the two consecutive quasi-derivatives $L_{q-1} v, L_{q} v$ are of the same sign, say positive, on $[M, \infty)$, if such an index exists. Since $L_{q} v>0, L_{q-1} v$ increases, so by (4),

$$
\begin{aligned}
L_{q-2} v(x) & =L_{q-2} v(M)+\int_{M}^{x} L_{q-1} v / \rho_{q-1} d x \\
& \geqslant L_{q-2} v(M)+L_{q-1} v(M) \int_{M}^{x} d x / \rho_{q-1} \rightarrow+\infty
\end{aligned}
$$

as $x \rightarrow \infty$. Since $L_{q-2} v$ does not vanish on $[M, \infty)$, it is necessarily positive there. Similarly, we have $L_{t} v>0, t=0, \ldots, q, x \in[M, \infty)$. Since $q$ is the greatest index with the above property, $(-1)^{t-q} L_{t} v>0, t=q, \ldots, n, x \in$ $[M, \infty) . L_{t} y\left(x, s_{i_{j}}\right) \rightarrow L_{t} v$ as $s_{i_{j}} \rightarrow \infty$, hence, for sufficiently large $s_{i, j}$,

$$
\begin{aligned}
L_{t} y\left(M, s_{i j}\right)>0, & t & =0, \ldots, q, \\
(-1)^{t-q} L_{t} y\left(M, s_{i j}\right)>0, & t & =q, \ldots, n .
\end{aligned}
$$

We prove that $q=k$. Assume that $L_{0} y=\rho_{0} y\left(x, s_{i_{j}}\right)$ has $l(l \geqslant 0)$ simple 
zeros in $(c, M)$, say $\alpha_{1}, \ldots, \alpha_{l}$. By (7), $L_{1} y$ has at least $l$ simple zeros in (c, $M)$, one in each of the intervals $\left(c, \alpha_{1}\right),\left(\alpha_{1}, \alpha_{2}\right), \ldots,\left(\alpha_{l-1}, \alpha_{l}\right)$. Similarly, we obtain that $L_{k-1} y$ has at least $l$ zeros in $(c, M)$, say $\beta_{1}, \ldots, \beta_{l} . L_{k} y$ has at least one simple zero in each of the intervals $\left(c, \beta_{1}\right), \ldots,\left(\beta_{l-1}, \beta_{l}\right)$. But if $q \leqslant k-1$, then $L_{k} y$ has an additional simple zero in $\left(\beta_{l}, M\right)$. For, if $L_{k} y$ has a fixed sign on $\left(\beta_{l}, M\right)$, then by $(10)$ we have $\operatorname{sgn}\left[L_{k} y\right]=-\operatorname{sgn}\left[L_{k-1} y\right]$ on $\left(\beta_{l}, M\right)$ and

$$
L_{k-1} y\left(\beta_{l}\right)=L_{k-1} y(M)-\int_{\beta_{l}}^{M} L_{k} y / \rho_{k} d x \neq 0,
$$

which is impossible. Thus $L_{k} y$ has at least $l+1$ zeros in $(c, M)$. By repeated use of (10), we obtain that $L_{n} y$ also changes its sign at least $l+1$ times in (c, $M)$, i.e., more than $y$. This contradicts (2), so $q \geqslant k$.

To obtain an upper bound for $q$, we consider $y\left(x, s_{i}\right)$ in $\left[M, s_{i}\right]$. If for a certain $t, 0 \leqslant t \leqslant q-1$, we denote the first zero of $L_{t} y$ in $\left(M, s_{i j}\right.$ ] by $\gamma$, then $L_{t+1} y$ has a simple zero in $(M, \gamma)$. Otherwise, by (9), $L_{t} y, L_{t+1} y$ would be positive on $(M, \gamma)$ and $L_{t} y(\gamma)=L_{t} y(M)+\int_{M}^{\gamma} L_{t+1} y / \rho_{t+1} d x>0$. By the $q+1$ conditions of (9), we may repeat this argument $q$ times. Let $y$ have $l$ simple zeros in $\left(M, s_{i}\right)$. After $n$ differentiations we obtain by the above use of (9) and on account of the $n-k-1$ boundary conditions of (8) at $s_{i_{j}}$ that $L_{n} y$ has at least $l+q+(n-k-1)-n$ zeros in $\left(M, s_{i j}\right)$. Since $L_{n} y=-p y$, $q \leqslant k+1$.

We have proved that $k \leqslant q \leqslant k+1$. But by (9)-(10), $L_{n} y(M)=$ $(-1)^{n-q} L_{0} y(M)$, and so $(-1)^{n-q} p(x)<0$. Thus, by (6), $q$ and $k$ are of the same parity and so $q=k$. Therefore

$$
\begin{array}{rlrl}
L_{t} v & >0, & t & =0, \ldots, k, \\
(-1)^{t-k} L_{t} v>0, & t & =k, \ldots, n,
\end{array}
$$

on $[M, \infty)$.

Now we return to the conjugate points of (2). From the sequence $\left\{a_{i}\right\}$, $a_{i} \rightarrow \infty$, we choose an $a_{i}, a_{i} \geqslant M$, and denote it by $a$. Let $u(x)$ be the appropriate solution which satisfies

$$
\begin{aligned}
L_{t} u(a) & =0, & t & =0, \ldots, k-1, \\
L_{t} u(\eta(a)) & =0, & t & =0, \ldots, n-k-1,
\end{aligned}
$$

and let $u>0$ on $(a, \eta(a))$. We shall show that (11) and (12) are incompatible. (It can be shown that (11) is equivalent to the absence of a focal point in the sense of [9].)

$u$ has exactly $n$ zeros in $[a, \eta(a)]$, therefore $L_{t} u, t=0, \ldots, n-1$, has at least $n-t$ zeros in $[a, \eta(a)]$. Since $\rho_{n}\left(L_{n-1} u\right)^{\prime}=L_{n} u=-p u$ and $u>0$ on $(a, \eta(a))$, Rolle's theorem implies that $L_{t} u$ has exactly $n-t$ zeros in $[a, \eta(a)]$. Let $x_{t}$ be the first zero of $L_{t} u$ in $\left(a, \eta(a)\right.$ ]. Since $L_{n} u(\eta(a))=-p u(\eta(a))=0$, we define $x_{n}=\eta(a)$. Since $L_{t} u(a)=0, t=0, \ldots, k-1$, it follows that 


$$
a<x_{k}<x_{k-1}<\cdots<x_{1}<x_{0}=\eta(a) .
$$

For $t=k, \ldots, n-1, L_{t} u$ has exactly $n-t$ zeros, all in $(a, \eta(a)]$ and $L_{t+1} u$ has exactly $n-t-1$ zeros. Therefore $L_{t+1} u$ has in $(a, \eta(a))$ exactly one zero between two zeros of $L_{t} u$ and no other zeros. In particular, $x_{t+1}>x_{t}$ and we have

$$
a<x_{k}<x_{k+1}<\cdots<x_{n-1}<x_{n}=\eta(a) .
$$

Consider the solution $w_{\lambda}=v-\lambda u$. For $\lambda=0, L_{t} w_{0}=L_{t} v \neq 0$ on $[M$, $\infty) \supset[a, \eta(a)]$. Let $\lambda_{0}$ be the smallest positive value of $\lambda$, such that for some $t$, $0 \leqslant t \leqslant n-1, L_{t} w_{\lambda}$ vanishes in $\left[a, x_{t}\right]$. No quasi-derivative $L_{t} w_{\lambda_{0}}$ changes its sign on $\left(a, x_{t}\right)$. Otherwise, by continuity, $L_{t} w_{\lambda}$ would change its sign for $\lambda$ sufficiently close to $\lambda_{0}$, contradicting the definition of $\lambda_{0}$. Therefore

$$
\begin{aligned}
L_{t} w_{\lambda_{0}} \geqslant 0, & x \in\left[a, x_{t}\right], t=0, \ldots, k, \\
(-1)^{t-k} L_{t} w_{\lambda_{0}} \geqslant 0, & x \in\left[a, x_{t}\right], t=k, \ldots, n,
\end{aligned}
$$

and at the endpoints

$$
\begin{aligned}
L_{t} w_{\lambda_{0}}(a) & =L_{t} v(a)>0, & & t=0, \ldots, k-1, \\
(-1)^{t-k} L_{t} w_{\lambda_{0}}\left(x_{t}\right) & =(-1)^{t-k} L_{t} v\left(x_{t}\right)>0, & & t=k, \ldots, n-1 .
\end{aligned}
$$

We shall prove that (15) and (16) imply that no $L_{t} w_{\lambda_{0}}, 0 \leqslant t \leqslant n-1$, has a zero in $\left[a, x_{t}\right]$, and this contradicts the definition of $\lambda_{0}$. For $k \leqslant t \leqslant n-1$, $(-1)^{t-k} L_{t} w_{\lambda_{0}}\left(x_{t}\right)>0$ and $(-1)^{t-k} L_{t+1} w_{\lambda_{0}} \leqslant 0$ on $\left[a, x_{t+1}\right]$. Since, by (14) $a<x_{t}<x_{t+1},(-1)^{t-k} L_{t} w_{\lambda_{0}}$ is decreasing and nonvanishing on $\left[a, x_{t}\right]$.

For $0 \leqslant t \leqslant k-1$, we show that $L_{t+1} w_{\lambda_{0}} \geqslant 0$ on $\left[a, x_{t}\right]$. On $\left[a, x_{t+1}\right]$ $L_{t+1} w_{\lambda_{0}}$ is nonnegative by (15). As $x_{t+1}<x_{t}$ for $0 \leqslant t \leqslant k-1$, we consider $\left(x_{t+1}, x_{t}\right]$ separately. Since $u>0$ on $(a, \eta(a)), L_{t+1} u$ is positive on $\left(a, x_{t+1}\right)$ and changes its sign at $x_{t+1}$. Therefore, on $\left(x_{t+1}, x_{t}\right], L_{t+1} u<0$ and we have $L_{t+1} w_{\lambda_{0}}=L_{t+1} v-\lambda_{0} L_{t+1} u>0$. Since $L_{t} w_{\lambda_{0}}(a)>0$, and $L_{t+1} w_{\lambda_{0}} \geqslant 0$ on [a, $\left.x_{t}\right], L_{t} w_{\lambda_{0}}$ increases and has no zeros on $\left[a, x_{t}\right]$.

The last contradiction proves that (11) and (12) are incompatible, and so our assumption that $y(x, s)$ has only a finite number of bounded zeros as $s \rightarrow \infty$, is false. Therefore, as $s \rightarrow \infty$, the number of zeros of $y(x, s)$ in $(c, s)$ increases indefinitely, and all of them are bounded from above. Since all these zeros are also bounded from below by $c$, the solution $\lim _{s \rightarrow \infty} y(x, s)$ has infinitely many zeros in $[c, \infty)$. This completes the proof of Theorem 1 .

Proof of Theorem 2. Theorem 1 proves the implication $(2) \rightarrow(1)$. It also proves $(2) \rightarrow(3)$, because the solution $\lim _{s \rightarrow \infty} y(x, s)$ vanishes at $c$ and has infinitely many zeros on $[c, \infty)$. (1) $\rightarrow(2)$ follows by the definition of $\eta(a)$ and (3) $\rightarrow(2)$ is almost trivial. Assume that $\eta_{i}(c)$ exists for every $i$ and let $y_{i}(x)$ have $n-1+i$ zeros in $\left[c, \eta_{i}(c)\right]$. Let $a$ be an arbitrary point, $a>c$. Since (2) has continuous coefficients, the number of zeros of any solution, in particular $y_{i}(x)$, on $[c, a]$ is bounded by an absolute constant. So, for sufficiently large $i$, $y_{i}(x)$ has at least $n$ zeros in $\left[a, \eta_{i}(c)\right]$ and $\eta(a)$ exists. 
Theorem 1 enables us to use results about disconjugacy for problems of nonoscillation. For example,

Corollary 1. Let $P(x) \geqslant p(x)>0$ or $P(x) \leqslant p(x)<0$. If the equation

$$
L_{n} y+P(x) y=0
$$

is nonoscillatory, then also (2) is nonoscillatory.

This follows from the well-known fact that $\eta(a, p)>\eta(a, P)$ [6], [8].

Since a differential equation is disconjugate if and only if its adjoint is disconjugate [8], their first conjugate points are equal and we have:

COROllary 2. Equation (2) is nonoscillatory if and only if its adjoint is nonoscillatory.

\section{REFERENCES}

1. U. Elias, The extremal solutions of the equation $L y+p(x) y=0$. II, J. Math. Anal. Appl. 55 (1976), 253-265.

2. __ Eigenvalue problems for the equation $L y+\lambda p(x) y=0$, (preprint).

3. M. S. Keener, On the equivalence of oscillation and the existence of infinitely many conjugate points, Rocky Mountain J. Math. 5 (1975), 125-134.

4. G. B. Gustafson, The nonequivalence of oscillation and nondisconjugacy, Proc. Amer. Math. Soc. 25 (1970), 254-260.

5. W. Leighton and Z. Nehari, On the oscillation of solutions of selfadjoint linear differential equations of the fourth order, Trans. Amer. Math. Soc. 89 (1958), 325-377.

6. A. J. Levin, Some questions bearing on the oscillation of solutions of linear differential equations, Soviet. Math. Dokl. 4 (1963), 121-124.

7. Z. Nehari, Nonoscillation criteria for nth order linear differential equations, Duke Math. J. 32 (1965), 607-616.

8. _ Disconjugate linear differential operators, Trans. Amer. Math. Soc. 129 (1967), $500-516$.

9. __ Green's functions and disconjugacy, Arch. Rational Mech. Anal. 62 (1976), 53-76.

10. J. R. Ridenhour, Linear differential equations where nonoscillation is equivalent to eventual disconjugacy, Proc. Amer. Math. Soc. 49 (1975), 366-372.

11. W. F. Trench, Canonical forms and principal systems for general disconjugate equations, Trans. Amer. Math. Soc. 189 (1974), 319-327.

Department of Mathematics, Technion, I. I. T., Haifa, Israel

Current address: Department of Mathematics, Carnegie-Mellon University, Pittsburgh, Pennsylvania 15213 